\title{
Reconstitution and use of highly active human CDK1:Cyclin-B:CKS1 complexes
}

Pim J. Huis in 't Veld ${ }^{1, \#}$, Sabine Wohlgemuth ${ }^{1}$, Carolin Koerner, ${ }^{1}$, Franziska Mueller', Petra Janning $^{2}$, Andrea Musacchio ${ }^{1,3, \#}$

\author{
1. Department of Mechanistic Cell Biology, Max Planck Institute of Molecular Physiology, Dortmund, Germany \\ 2. Department of Chemical Biology, Max Planck Institute of Molecular Physiology, Dortmund, Germany \\ 3. Centre for Medical Biotechnology, Faculty of Biology, University Duisburg-Essen, Universitätsstrasse 45141 Essen, Germany.
}

\author{
\#Corresponding authors \\ pim.huis@mpi-dortmund.mpg.de; andrea.musacchio@mpi-dortmund.mpg.de;
}

\begin{abstract}
As dividing cells transition into mitosis, hundreds of proteins are phosphorylated by a complex of cyclin-dependent kinase 1 (CDK1) and Cyclin-B, often at multiple sites. CDK1:Cyclin-B phosphorylation patterns alter conformations, interaction partners, and enzymatic activities and need to be recapitulated in vitro for the structural and functional characterization of the mitotic protein machinery. This requires a pure and active recombinant kinase complex. The kinase activity of CDK1 critically depends on the phosphorylation of a Threonine residue in its activation loop by a CDK1 activating kinase (CAK). We developed protocols to activate CDK1:Cyclin-B either in vitro with purified CDK1 activating kinases (CAK) or in insect cells through CDK-CAK coexpression. To boost kinase processivity, we reconstituted a tripartite complex consisting of CDK1, Cyclin-B, and CKS1. In this work, we provide and compare detailed protocols to obtain and use highly active CDK1:Cyclin-B (CC) and CDK1:Cyclin-B:CKS1 (CCC).
\end{abstract}




\section{Introduction}

A human cell that sets out to divide needs to condense DNA into chromosomes, break down the nuclear envelope, and form a mitotic spindle. Key for the initiation of these transformations is the

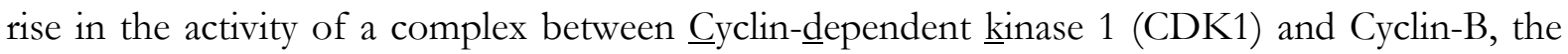
main mitotic cyclin in vertebrates (Morgan, 2007; Hochegger et al., 2008). This results in the posttranslational modification of hundreds of proteins, including other mitotic kinases, on specific serine and threonine side-chains and often at multiple sites (Daub et al., 2008; Olsen et al., 2010; Hegemann et al., 2011). Tight spatiotemporal regulation of these substrates and of their role in mitosis is then enabled by the intricate balance between mitotic kinase activities and counteracting phosphatases (Gelens et al., 2018).

Phosphorylation regulates the conformation, the activity, and the binding partners of the mitotic protein machinery. CDK1 is primed to initiate mitosis after Cyclin-B replaces Cyclin-A as the major CDK1-associated Cyclin and after the side-chains of Thr14 and Tyr15 in CDK1 are dephosphorylated. This is promoted by a shift in balance between the kinases Wee1 and Myt1 and the Cdc25 phosphatase, and ultimately discharges the glycine-rich loop (GEGTYG) from antagonizing substrate-binding and ATP hydrolysis (Morgan, 2007; Wood and Endicott, 2018). The activity of CDK kinases also critically depends on the configuration of its activation loop. The phosphorylation of this loop, at position Thr161 in CDK1, is required to dock the activation loop at the base of the active site, enabling efficient substrate binding and phosphate transfer (Jeffrey et al., 1995; Russo et al., 1996; Brown et al., 2015) (Figure 1A, 1B). Active CDK1 is thus phosphorylated at Thr161 and dephosphorylated at positions Thr14 and Tyr15.

Phosphorylation of the activation loop of CDK1 occurs in trans by a $\underline{C D K}$-activating kinase (CAK). In metazoans, CDK1 is activated by the CDK7:Cyclin-H kinase(Fisher and Morgan, 1994; Larochelle et al., 1998), which is also implicated, as a module of TFIIH, in the phosphorylation of the C-terminal domain of RNA polymerase II, as recently depicted (Greber et al., 2020; Abdella et 
al., 2021; Chen et al., 2021). Whereas metazoan CAK consists of a CDK7:Cyclin-H complex, activation of the budding yeast CDK1 (Cdc28) is mediated by the distantly related and monomeric CAK1 (hereafter scCAK1)(Espinoza et al., 1996; Kaldis et al., 1996; Thuret et al., 1996).

Besides phosphorylation, the CDK1:Cyclin-B kinase activity and substrate binding preferences are also regulated by various binding proteins. An important regulator is CKS1, a small adaptor protein that binds to the large helical lobe of the CDK1 kinase fold (Figure 1C). CKS1 contains a binding pocket for phosphorylated threonine residues and guides substrates with proximal phosphothreonine residues to the active site of CDK1, resulting in multisite phosphorylation (Kõivomägi et al., 2011, 2013).

With a persistent interest in the functional and structural characterization of proteins that act in cell division, and attempts to reconstitute in vitro fundamental aspects of the cell division process, it is essential to recapitulate CDK1:Cyclin-B phosphorylation patterns in vitro with a recombinant kinase complex that is pure, active, and processive. We have developed and compared ways to activate recombinant CDK1 with yeast or human CAK in vitro or in insect cells during protein expression. To boost the processivity of reconstituted CDK1:Cyclin-B complexes, we also set out to include CKS1 in our recombinant kinase complexes. Taken together, our analysis demonstrates how to reconstitute CDK1:Cyclin-B (CC) and CDK1:Cyclin-B:CKS1 (CCC) complexes, how to phosphorylate the activation loop of CDK1 efficiently, and how to reconstitute multi-site CDK1 phosphorylation patterns in vitro. 


\section{Results}

\section{Reconstitution of stoichiometric CDK1:Cyclin-B and CDK1:Cyclin-B:CKS1 complexes}

We set out to reconstitute CDK1:Cyclin-B (CC) and CDK1:Cyclin-B:CKS1 (CCC) complexes from their purified individual constituents. We therefore expressed full-length CDK1, Cyclin-B1, and CKS1 in insect cells and purified these proteins to homogeneity using size-exclusion chromatography after affinity tags were proteolytically removed (Figure 1D). Stable and stoichiometric CC (83 kDa) and CCC $(93 \mathrm{kDa})$ complexes formed when CDK1, Cyclin-B, and CKS1 were mixed and were further purified using size-exclusion chromatography (Figure 1E). These results highlights that the formation of CDK1:Cyclin-B or CDK1:Cyclin-B:CKS1 complexes does not require the phosphorylation of the CDK1 activation loop (see below).

\section{Phosphorylation of CDK1 in insect cells and in vitro by a CDK activating kinase}

Since the phosphorylation of $\mathrm{CDK} 1^{\mathrm{Thr} 161}$ by the $\underline{\mathrm{CDK}}$ activating kinase (CAK) promotes substrate binding and phosphorylation, we set out to co-express CDK1 and CAK in insect cells. Purified CDK1 that was co-expressed with either yeast or human CAK (scCAK1 or hsCDK7, respectively) was effectively phosphorylated, as judged by its retarded mobility on a phostag-containing acrylamide gel (Kinoshita et al., 2006) (Figure 2, lanes 1-3). We also purified scCAK1 (39 kDa) and used it to phosphorylate CDK1 in vitro. Analysis by phostag-PAGE showed effective phosphorylation of CDK1 in a reaction of 100 minutes at room temperature (Figure 2, lanes 48). CDK1 associated with Cyclin-B is also a CAK substrate, but the rate of phosphorylation was slower when CDK1 was in the Cyclin-bound state (Figure 2, lanes 4-13). The similar migration of Cyclin-B throughout the course of the experiment confirms that Cyclin-B is neither a CDK nor a CAK substrate. 


\section{Phosphorylation of CDK1 Threonine 161 activates recombinant CDK1:Cyclin-B}

We next used semi-quantitative mass spectrometry to assess if the CAK-induced shift in CDK's phostag-PAGE migration reflected phosphorylation of Threonine 161. Analysis of CDK1 that was exposed to human or yeast CAK in insect cells or in vitro confirmed the efficient phosphorylation of Threonine 161 and demonstrated that very few additional CDK1 residues, if any, were phosphorylated, and grossly substoichiometrically (Supplementary Table 1). Exposure to lambda-phosphatase reversed Thr161 phosphorylation (Supplementary Figure 1). The overall degree of Thr161 phosphorylation detected by mass spectrometry matches the intensities of the modified and unmodified CDK on phostag-PAGE (Figure 3A-D, Supplementary Figure 1). Whereas CDK1 was efficiently phosphorylated by scCAK1 both in vitro (92\%) and upon coexpression in insect cells (91\%), the phosphorylation by hsCDK7 was markedly lower in vitro than in insect cells (22\% vs 78\%) (Figure 3A-D, compare reactions 2, 3, 5, 7, and Supplementary Figure 1).

To test and compare the activity of CDK1:Cyclin-B complexes, we used a 373-residue fragment encompassing the $\mathrm{N}$-terminal region of the kinetochore protein CENP-T. This is an ideal multisite phosphorylation model substrate as it is predominantly disordered and is phosphorylated by CDK1:Cyclin-B on multiple sites in vivo and in vitro (Nishino et al., 2013; Kim and Yu, 2015; Rago et al., 2015; Huis in 't Veld et al., 2016). CENP-T $T^{1-373}$, expressed in bacterial and therefore unphosphorylated, was initially purified, labelled at its carboxy-end with tetramethylrhodamine (TMR) using Sortase-mediated conjugation (Guimaraes et al., 2013; Hirakawa et al., 2015), and finally purified to homogeneity using size-exclusion chromatography.

We used CENP-T at a concentration of $10 \mu \mathrm{M}$ and varied the concentration of the CDK1:CyclinB complexes between $20 \mathrm{nM}$ and $2 \mu \mathrm{M}$. To monitor its phosphorylation, we used in-gel fluorescence after phostag-PAGE (Figure 3E, F). CENP-T was not phosphorylated after 90 minutes in the absence of kinase or when incubated with CDK1 without Cyclin-B, highlighting 
Figure 1: Reconstitution of stoichiometric CDK1:Cyclin-B and CDK1:Cyclin-B:CKS1 complexes

a) Surface view of a complex between CDK1 (green) and Cyclin-B (purple) (PDB 4YC3)(Brown et al., 2015). The CDK1 162-173 activation loop is shown in gold and Threonine 161 in orange. Within the closed activation loop, residues CDK1 162-164 (HEV) are not visible and replaced with a dashed line. $\mathbf{b})$ Surface view of CDK2:Cyclin-A with bound ATP (PDB 1JST)(Russo et al., 1996). The side chains of Arginines 50, 126, and 150 coordinate the activation loop with a phosphorylated Threonine 160. c) Surface view of a tripartite CDK1:Cyclin-B:CKS1 complex (PDB 4YC3). Compared to panel a), the structure is rotated $45^{\circ}$ along the x-axis. d, e) Analysis of purified CDK1, Cyclin-B, CKS1, CDK1:Cyclin-B (CC), and CDK1:CyclinB:CKS1 (CCC) by SDS-PAGE followed by Coomassie staining and size exclusion chromatography using a Superdex 200 increase 5/150 column.
A

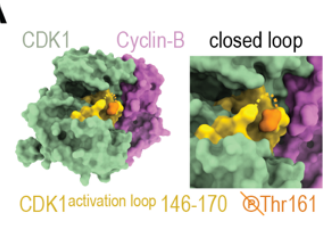

B

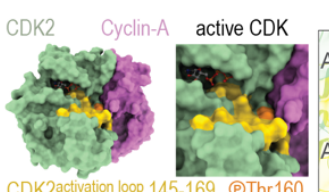

C

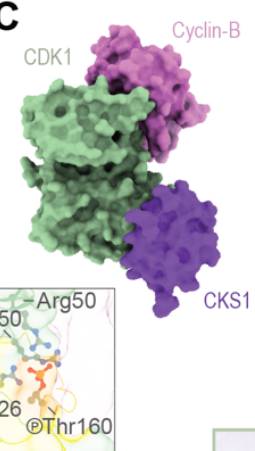

D 0 atcing $-150$ -75
$-\quad-50$ $-37$ $-37$ $-25$ $-20$ $--15$

E 300
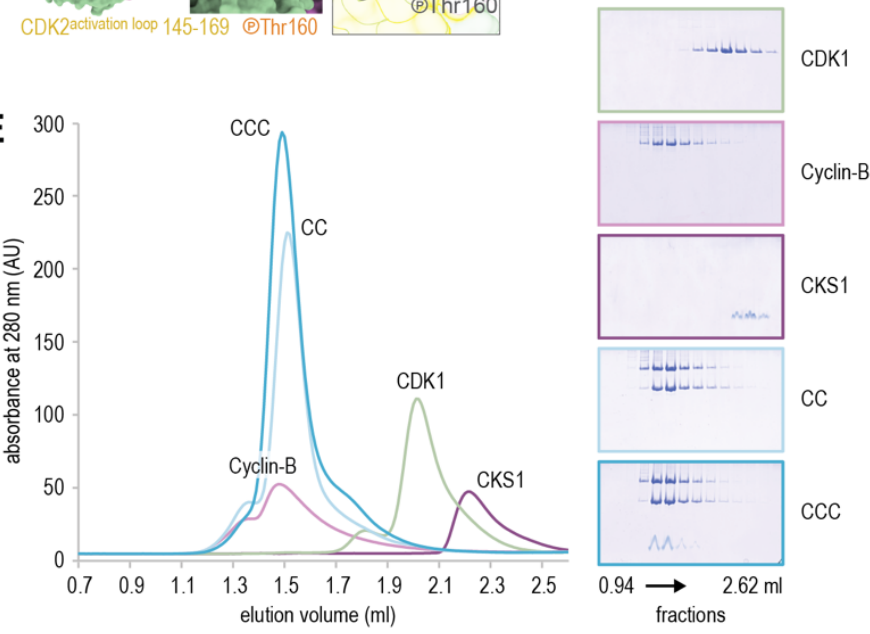

Figure 2: Phosphorylation of CDK1 in insect cells and in vitro by a CDK activating kinase

a) Reaction scheme for the phosphorylation of CDK1 with scCAK1. b, c) SDS-PAGE analysis of CDK1, Cyclin-B, and scCAK1. The presence of phostag-acrylamide (panel c) slows the migration of phosphorylated CDK1.
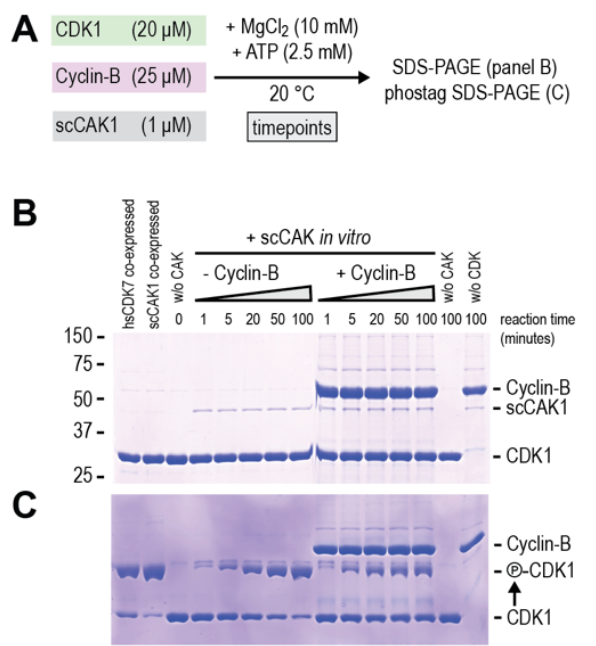
the purity of the CENP-T and CDK1 samples and the strict Cyclin-dependency of CDK1 activity (Figure 3F, reactions 16 and 7). Sparse CENP-T phosphorylation occurred in the absence of CDK1, probably caused by traces of kinase in the Cyclin-B sample (Figure 3F, reactions 14 and 15). Relative to this control sample, phosphorylation appeared enhanced when CENP-T was incubated with CDK1:Cyclin-B that had not been exposed to a CAK. At CDK1:Cyclin-B concentrations of 0.4 or $2 \mu \mathrm{M}$, CENP-T underwent a progressive increase in phosphorylation between 15 and 90 minutes (Figure 3F, reactions 1, 4, 8, and 11). By contrast, apparently homogeneous multi-site CENP-T phosphorylation patterns were already visible after 15 minutes of phosphorylation with 0.2 or $0.4 \mu \mathrm{M}$ CDK1:Cyclin-B complexes generated with kinase coexpressed with scCAK1 or hsCDK7 in insect cells (Figure 3F, reactions 2, 3, and 21). After a 90minute reaction, comparable CENP-T phosphorylation levels were observed with concentrations of $20 \mathrm{nM}$ or $2 \mu \mathrm{M}$ of CAK-treated or CAK-untreated CDK1:Cyclin-B complexes, respectively (Figure 3F, reactions 8, 11, and 20). CAK exposure thus increases the activity of recombinant CDK1:Cyclin-B complexes on CENP-T by approximately two orders of magnitude. Consistent with a modest but marked increase in the phosphorylation levels of CDK1 Thr161, the in vitro activation of CDK1:Cyclin-B with hsCDK7 resulted in increased CENP-T phosphorylation (Figure 3F, reactions 4 and 5). However, CDK1 activation in vitro with scCAK1 was more effective (Figure 3F, reactions 5 and 6), indicating that the phosphorylation of Thr161 correlates with kinase activity.

\section{CKS1 boosts the processivity of CDK1:Cyclin-B}

A side-by-side comparison of pre-assembled CDK1:Cyclin-B (CC) and CDK1:Cyclin-B:CKS1 (CCC) complexes suggested the existence of a slower migrating form of CENP-T in the CCC reactions (Figure 3F, reactions 18, 19, 21, and 22). To investigate putative effects of CKS1 on CDK1:Cyclin-B processivity, we followed the phosphorylation of CENP-T with $100 \mathrm{nM}$ of CC 
bioRxiv preprint doi: https://doi.org/10.1101/2021.09.24.461690; this version posted September 24, 2021 . The copyright holder for this preprint (which was not certified by peer review) is the author/funder, who has granted bioRxiv a license to display the preprint in perpetuity. It is made available under aCC-BY-NC 4.0 International license.

or CCC over time (Figure 4A, B). These complexes contain CDK1 that was activated by scCAK1 co-expression and were reconstituted from individual constituents with (pre-assembled) or without (in vitro assembly) size-exclusion chromatography (Figure 4A, B).

Following these reactions over time revealed that the presence of CKS1 sped up CENP-T phosphorylation approximately two-fold (Figure 4C, D). In addition to faster phosphorylation, CENP-T migration appeared to continuously decrease over time in the presence of CCC. This suggests that low-affinity CENP-T sites become CDK1 substrates when proximal phosphorylated threonine residues dock to CKS1 (Kõivomägi et al., 2011, 2013). The difference between high $(\sim 5-10$ sites $)$ and hyper $(10+$ sites $)$ phosphorylation on CENP-T was most apparent when the phostag concentration in the polyacrylamide gel was lowered five-fold (Figure 4E). Taken together, we demonstrate that the presence of CKS1 enables CDK1:Cyclin-B to efficiently establish multi-site phosphorylation patterns. 
bioRxiv preprint doi: https://doi.org/10.1101/2021.09.24.461690; this version posted September 24, 2021. The copyright holder for this

preprint (which was not certified by peer review) is the author/funder, who has granted bioRxiv a license to display the preprint in perpetuity. It is made available under aCC-BY-NC 4.0 International license.

\section{Figure 3: Phosphorylation of CDK1 Threonine 161 activates recombinant CDK1:Cyclin-B}

a, b) Preparation and SDS-PAGE analysis of CDK1:Cyclin-B complexes that were either assembled from purified components or pre-assembled into dimeric or trimeric complexes. Reactions 1 and 4 are technical replicates. c, d) Selected CDK1:Cyclin-B complexes were analyzed for phosphorylation by mass spectrometry (technical triplicates) and phostag SDS-PAGE. Chart diagrams show the summed average intensities of all phosphorylated (green) and nonphosphorylated (grey) peptides containing Thr161. See Supplementary figure 1 for more information. The effect of scCAK1 or hsCDK7 co-expression on the phostag-migration of CDK1 is shown in Figure 2c, lanes 1-3. e, $\mathbf{f}$ ) Fluorescently labeled CENP-T was exposed to different kinase complexes and analysed for multi-site phosphorylation using phostag SDS-PAGE. The same gels were analysed for in-gel fluorescence (CENP-T) and Coomassie staining (all proteins). Reactions 1 and 4 as well as 8 and 11 are technical replicates.
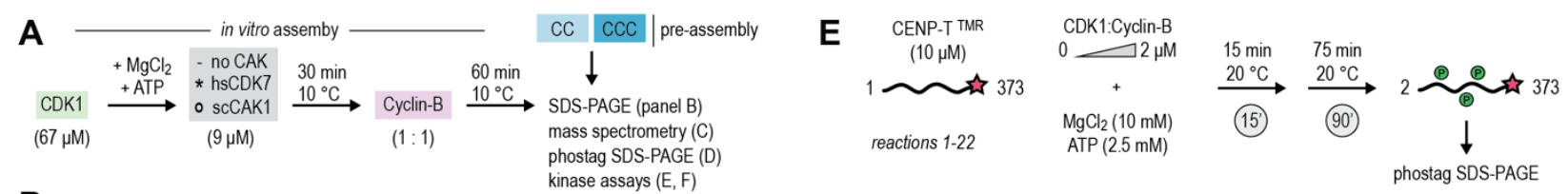

B
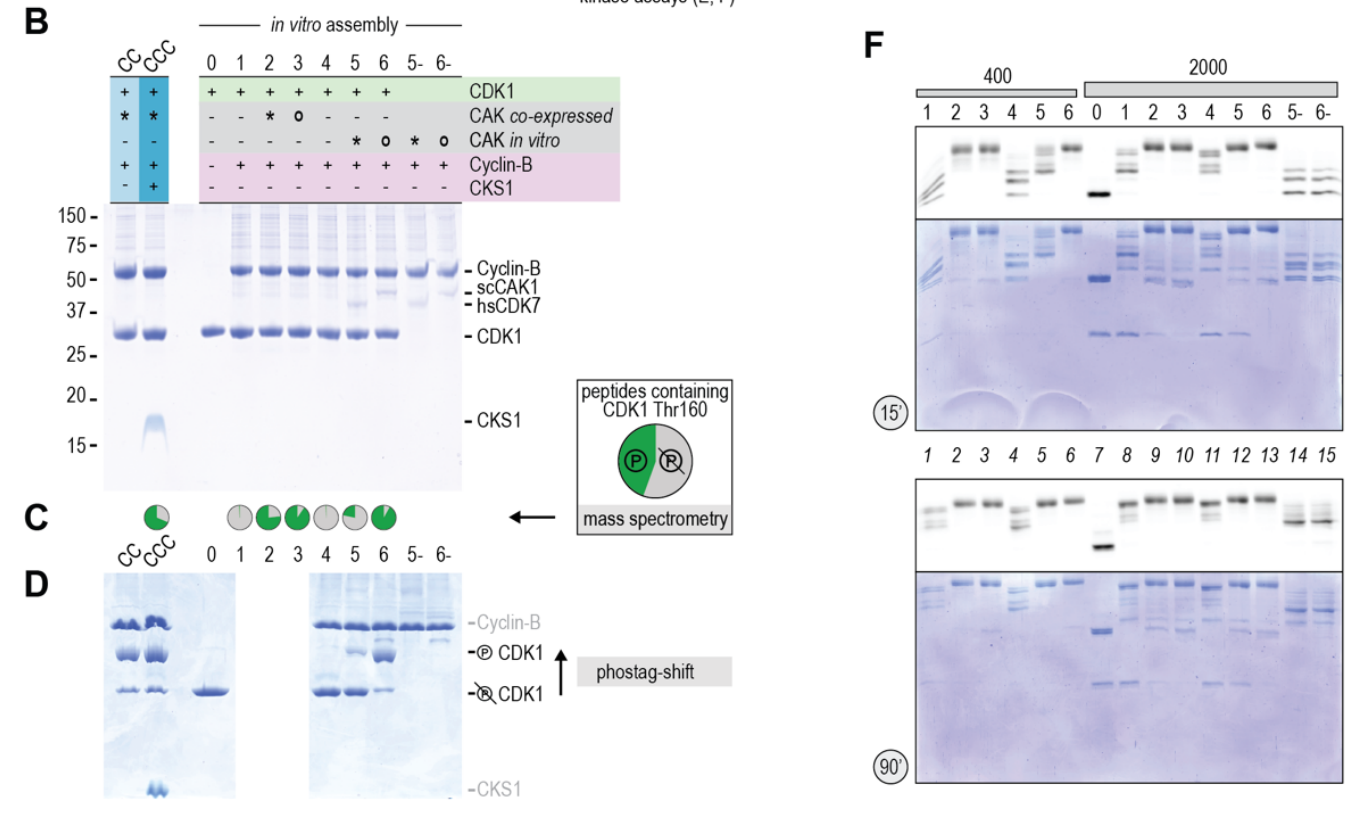

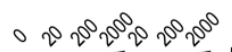

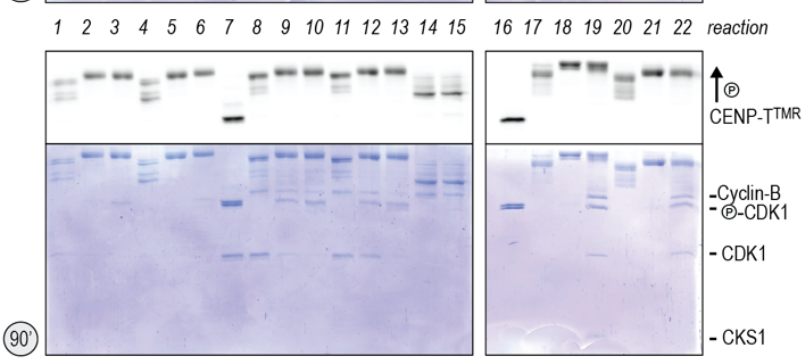


bioRxiv preprint doi: https://doi.org/10.1101/2021.09.24.461690; this version posted September 24, 2021. The copyright holder for this

preprint (which was not certified by peer review) is the author/funder, who has granted bioRxiv a license to display the preprint in perpetuity. It is made available under aCC-BY-NC 4.0 International license.

Figure 4: CKS1 boosts the processivity of CDK1:Cyclin-B

a) Preparation and phostag SDS-PAGE analysis of CDK1:Cyclin-B and CDK1:Cyclin-B:CKS1 complexes that were either assembled from purified components or pre-assembled into dimeric or trimeric complexes. b, c) CENP-T was exposed to different kinase complexes and analysed for multi-site phosphorylation using phostag SDS-PAGE and ingel fluorescence (CENP-TTMR). d) Quantification of phosphorylated / non-phosphorylated signals from the gels shown in panel c). e) SDS-PAGE analysis of samples CC (3) and CCC (4) as in panel c) in the presence of $10 \mu \mathrm{M}$ phostag acrylamide (all other gels contain $50 \mu \mathrm{M}$ phostag-acrylamide).

A

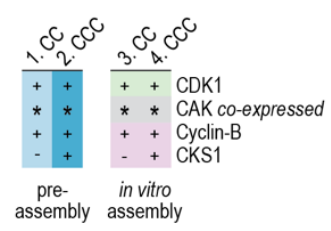

B

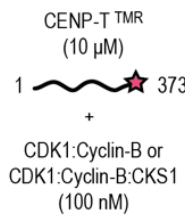

$(100 \mathrm{nM})$

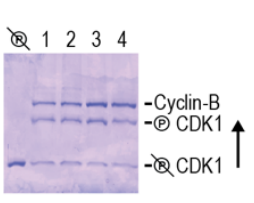

CENP-T TMR

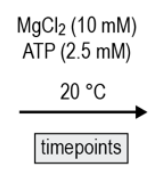

timepoints

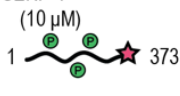

phostag SDS-PAGE
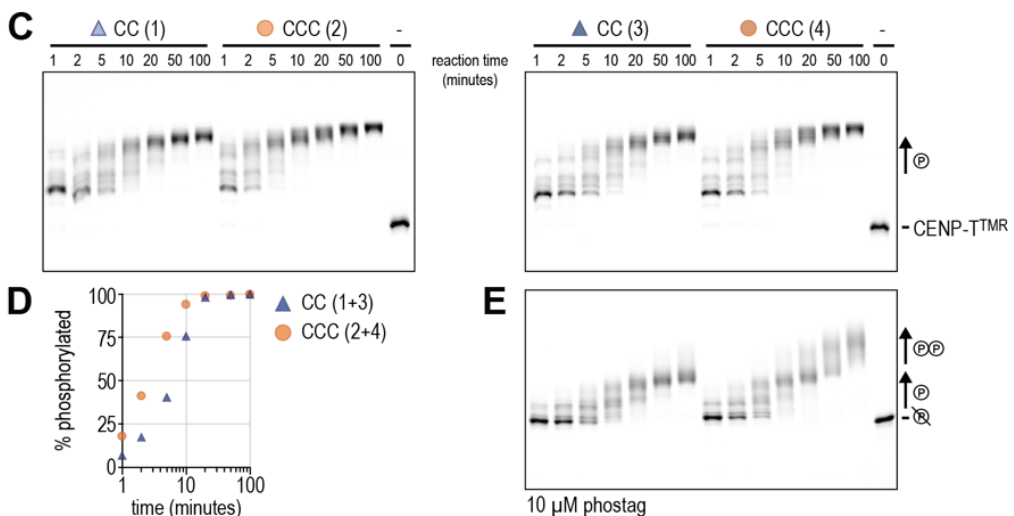

Supplementary Figure 1: Analysis of phosphorylation of CDK1's Threonine 160 by mass spectrometry

a) Overview of samples analyzed by mass spectrometry. With the exception of CCC- $\lambda$, all samples were also included in Figure 3.

b) Five different peptides containing CDK1 Thr 161 were detected after trypsin digestion. From three technical replicates, the average summed intensities (AU) were added for peptides with phosphorylated Thr 161 and for peptides with nonphosphorylated Thr 161. Intensities of phosphopeptides as a fraction of the total peptide peptide intensities are shown in the last column.
A

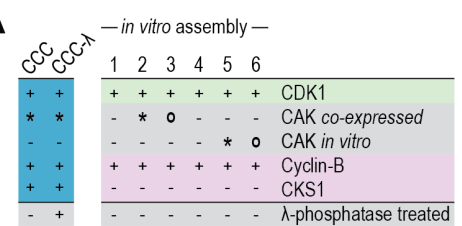

B

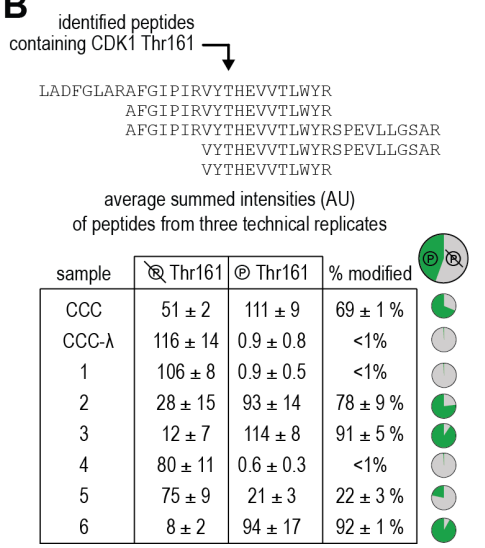




\section{Discussion}

Over the last decades, genetic, biochemical, and structural studies provided detailed insights into the regulation of the cell cycle by CDK:Cyclin combinations and the activation of Cyclindependent Kinases by CAKs (Morgan, 2007; Wood and Endicott, 2018). Since hundreds of CDK:Cyclin phosphorylation events are required to rewire the protein machinery in dividing mitotic or meiotic cells, in vitro reconstitution of phosphorylation patterns for the functional and structural characterization of simplified, smaller subsets of components is mandatory. To facilitate this type of experiments, the availability of pure and active recombinant CDK1:Cyclin-B complexes is essential, and here we have contributed to the development of a strong pipeline towards this goal. A previous study demonstrated how to obtain a pure and active CDK1:CyclinB complex using purified scCAK1 (expressed in bacteria) and CDK1 (expressed in insect cells), in vitro phosphorylation of CDK1 with scCAK1, and subsequent assembly of the active CDK1:Cyclin-B complex (Brown et al., 2015). In this study, we built on this previous work and took a systematic approach to optimize protocols for the generation of highly active and processive recombinant CDK1:Cyclin-B:CKS1 complexes. These robust and easy-to-implement protocols and the corresponding expression plasmids, publicly available through Addgene (https://www.addgene.org/), will be a valuable resource for researchers with a range of backgrounds and an interest in investigating the protein machinery orchestrating cell division. We demonstrate how in-gel fluorescence and general protein staining after phostag-PAGE is a costeffective and swift combination to analyze single and multi-site phosphorylation. Whereas mass spectrometry and site-directed mutagenesis of putative phosphorylation sites are needed to specify modified residues, phostag-PAGE provides a clear overview of phosphorylation coverage -for example to follow the amounts of phosphorylated and non-phosphorylated CDK1 Thr161- and can readily be combined with immunofluorescence. The latter is especially useful to study more complex reaction mixtures. 
Co-expression of CDK1, Cyclin-B, and CKS1 in insect cells was used in a very recent study that revealed how recombinant CCC complexes form a stoichiometric complex with Separase (Yu et al., 2021). In that study, the CCC assembly complexed to Separase had been obtained by insect cell co-expression, but had not been co-expressed with CAK or treated with CAK activity during purification. Surprisingly, however, density for the phosphorylated Thr161 residues of CDK1 was clearly visible, demonstrating that at least a significant subset of CDK1 might have been targeted by an endogenous CAK activity in insect cells. This may seem to imply that monomeric CDK1, which we find to be very little $(<1 \%)$ phosphorylated on Thr161 when expressed as an isolated subunit without exogenous co-expression of CAK, is not a good substrate of the endogenous CAK activity of insect cells. This is consistent with previous studies describing that CDK7:CyclinH can phosphorylate CDK2 but not CDK1 in their Cyclin-free forms (Fisher and Morgan, 1994). Our finding that hsCDK7 is a potent CDK1 activator when co-expressed in insect cells but does not phosphorylate CDK1 efficiently in vitro also suggests that host-cell (ovary cells from the cabbage looper moth Trichopulsia ni) factors contribute to CAK activation that leads to the phosphorylation of CDK1's Thr161 (Figure 3). Such factors could include a hsCDK7 activating kinase (CAKAK) or a Cyclin that associates with hsCDK7 or CDK1. In fission yeast, it was for example demonstrated that the kinase Csk1 acts as a CAK and as a CAKAK (Hermand et al., 1998; Saiz and Fisher, 2002). In our work, we maximized robustness and yields by reconstituting CC or CCC complexes after individual expression of the constituents, and CDK1 was specifically activated through exposure to scCAK1 during co-expression or in vitro.

How multi-site phosphorylation rewires proteins that govern cell division was highlighted in a study showing that reconstituted APC/C could only bind its activator CDC20 after a region in the APC1 subunit was hyperphosphorylated and released from a CDC20 binding site on APC8 (Zhang et al., 2016). In that work, APC/C was phosphorylated in vitro with PLK1 kinase and a CDK2:Cyclin-A:CKS2 complex in a manner that reflects APC/C phosphorylation in mitosis 
(Kraft et al., 2003; Hegemann et al., 2011; Zhang et al., 2016). Although the constituents of the CDK2:Cyclin-A:CKS2 complex were individually expressed in bacteria and had not been exposed to a CAK, and were therefore most probably not phosphorylated, the resulting kinase did effectively reconstitute multi-site phosphorylation patterns on APC/C. CDK2:Cyclin-A:CKS2 has been previously shown to be strongly dependent on the phosphorylation of the activation loop in CDK2 (Hagopian et al., 2001). Thus, we speculate that the docking of CDK2:Cyclin-A:CKS2 on APC/C results in CAK-independent substrate phosphorylation. Supporting the latter explanation, we see that CDK1:Cyclin-B that had not been exposed to CAK efficiently phosphorylated CENP$\mathrm{T}$ at concentrations of 0.4 and $2 \mu \mathrm{M}$ in 90 minutes (Figure 3). Accordingly, we have used CDK1:Cyclin-B complexes that were not CAK-exposed at relatively high concentrations of 0.25 $1 \mu \mathrm{M}$ in recent studies to successfully phosphorylate substrates using reactions for 16 hours at $4^{\circ} \mathrm{C}$ (Huis in 't Veld et al., 2019; Piano et al., 2021; Singh et al., 2021).

In summary, we describe the discrete effects of activation loop phosphorylation and CKS binding on the activity and processivity of a recombinant CDK1:Cyclin B complex, together with protocols for expression and access to the relevant expression vectors. These tools will support work of in vitro reconstitution of crucial aspects of cell division. 


\section{Materials and Methods}

\section{Baculovirus generation and protein expression in insect cells}

CDK1, Cyclin-B1, CKS1B, and hsCDK7 cDNA constructs were codon-optimized for insect cell expression and obtained from GeneArt (Life Technologies). The scCAK1 (CIV1) construct was kindly provided by David Barford. Expression cassettes of CDK1, Cyclin-B, CKS1B, scCAK1, and hsCDK7 were inserted into pLIB vectors (Weissmann et al., 2016) with N-terminal GST-3C (CDK1) or Polyhistide-TEV (others) tags and baculoviruses were generated following standard protocols (Trowitzsch et al., 2010). For expression, $S f 9$ cells were infected for 3-4 days and added (1:20 dilution) to logarithmically growing Hi5-derived Tnao38 insect cells (Hashimoto et al., 2012). For co-expressions, Sf9 cultures expressing CDK1 (1:20 dilution) and scCAK1 or hsCDK7 (1:40 dilution) were simultaneously added to the expression culture. All insect cells were kept at $27^{\circ} \mathrm{C}$. Cells were harvested after 3 days by centrifugation at $1000 \mathrm{~g}$ at room temperature, washed once with ice-cold PBS, pelleted by centrifugation at $1000 \mathrm{~g}$ at $4{ }^{\circ} \mathrm{C}$, flash-frozen in liquid nitrogen, and stored at $-80{ }^{\circ} \mathrm{C}$.

\section{CDK1 purification}

All protein purification steps were performed on ice or at $4{ }^{\circ} \mathrm{C}$. Pellets from 2 liters of insect cells expressing GST-3C-CDK1 (with or without CAK co-expression) were resuspended in $160 \mathrm{ml}$ buffer A (50mM HEPES pH7.4, 250mNaCl, 2mM TCEP, 5\% v/v glycerol) supplemented with HP plus protease inhibitor mix (Serva) and DNase I (Roche). Cell lysates were prepared by sonication and cleared by centrifugation at $80000 \mathrm{~g}$ at $4^{\circ} \mathrm{C}$ for $30-45$ minutes. The soluble lysate was passed through a $0,8 \mu \mathrm{m}$ filter and loaded onto a column with $20 \mathrm{ml}$ Gluathione Sepharose 4 Fast Flow resin (Cytiva). After washing with 25 column volumes of buffer A, CDK1 was cleaved with in-house generated $3 \mathrm{C}$ protease for 16 hours. The eluate was concentrated to $2 \mathrm{ml}$ through centrifugation with a 30k Amicon filter (Millipore) and separated on a Superdex 200 16/600 
column equilibrated in buffer A. To remove GST, uncleaved GST-CDK1, and GST-3CPreScission, a $5 \mathrm{ml} \mathrm{GSH} \mathrm{column} \mathrm{(GE} \mathrm{Healthcare)} \mathrm{was} \mathrm{mounted} \mathrm{after} \mathrm{the} \mathrm{size-exclusion} \mathrm{column.}$ Selected fractions were concentrated to concentrations well above $100 \mu \mathrm{M}$. Purification from $2 \mathrm{l}$ of insect cells yielded approximately $4 \mathrm{mg}$ of CDK1.

\section{Cyclin-B purification}

His-TEV-Cyclin-B containing lysates were prepared as described above with $15 \mathrm{mM}$ imidazole in lysis and wash buffers. After loading onto a $5 \mathrm{ml}$ or $10 \mathrm{ml}$ Talon (Clontech) or Ni-NTA (GE Healthcare) column, and washing with approximately 50 column volumes, Cyclin-B was eluted in buffer A with $250 \mathrm{mM}$ imidazole and concentrated to $2 \mathrm{ml}$ through centrifugation with a $30 \mathrm{k}$ Amicon filter (Millipore). To remove the polyhistidine tag, Cyclin-B was exposed to TEV protease for 16 hours. Cyclin-B was further purified on a Superdex 200 16/600 column equilibrated in buffer A. To remove His-TEV protease and uncleaved His-Cyclin-B, a $5 \mathrm{ml}$ Talon column (GE Healthcare) was mounted after the size-exclusion column. Selected fractions were concentrated to concentrations well above $100 \mu \mathrm{M}$. Purification from 11 of insect cells yielded approximately 15 mg of Cyclin-B.

\section{CKS1 purification}

His-TEV-CKS1 was purified as described for Cyclin-B but using 5k Amicon filters and a Superdex 75 16/600 size-exclusion column. Approximately $5 \mathrm{mg}$ of CKS1 was obtained from 0.51 of insect cells.

\section{CDK1:Cyclin-B (CC) and CDK1:Cyclin-B:CKS1 (CCC) formation}

Purified CDK1, Cyclin-B, and CKS1 were mixed in a $1: 1: 0$ (CC) or $1: 1: 2$ (CCC) ratio for 1-2 hours on ice with concentrations above $100 \mu \mathrm{M}$. After size-exclusion chromatography on a Superdex 200 16/600 column in buffer A, fractions containing CC or CCC were concentrated 
through centrifugation with a 30k Amicon filter (Millipore), flash-frozen in liquid nitrogen, and stored at $-80^{\circ} \mathrm{C}$. Analytical size-exclusion chromatography (Figure 1) was performed using a Superdex 200 5/150 column.

\section{scCAK1 and hsCDK7 purification}

The CDK1 activating kinases scCAK1 and hsCDK7 were purified as described for Cyclin-B above and yielded approximately $0.5 \mathrm{mg}$ and $5 \mathrm{mg}$, respectively.

\section{CENP-T purification}

CENP-T ${ }^{2-373}$ was expressed, purified, and fluorescently labelled as described (Huis in 't Veld et al., 2016). In brief, the expression of GST-3C-CENP-T ${ }^{2-373}$ with a C-terminal -LPETGG extension was induced in E. coli BL21(DE3)-codon-plus-RIL cells through the addition of $0.35 \mathrm{mM}$ IPTG for $\sim 14$ hours at $20^{\circ} \mathrm{C}$. Subsequent steps were performed at $4^{\circ} \mathrm{C}$ or on ice. Cleared lysates were prepared by sonication and centrifugation and bound to a Glutathione-Agarose resin (Serva). GST-3C-CENP-T was cleaved off the beads with in-house generated 3C protease for 16 hours. After further purification using a Heparin HP column (GE Healthcare), CENP-T was fluorescently labeled with a GGGGK-TMR (5-Carboxytetramethylrhodamine) peptide (GenScript) using the Calcium-independent Sortase 7M (Hirakawa et al., 2015). After sizeexclusion chromatography using a Superdex 200 16/600 column (GE Healthcare) in a buffer containing $20 \mathrm{mM}$ Tris- $\mathrm{HCl}, \mathrm{pH} 8.0,150 \mathrm{mM} \mathrm{NaCl}$, and $1 \mathrm{mM}$ TCEP, CENP-T ${ }^{2-373}{ }^{\text {TMR }}$ was concentrated to $118 \mu \mathrm{M}$ and stored at $-80^{\circ} \mathrm{C}$.

\section{In vitro phosphorylation}

Phosphorylation reactions were performed in the presence of $\mathrm{MgCl}_{2}(10 \mathrm{mM})$ and ATP $(2.5 \mathrm{mM})$ and monitored on standard denaturing 10\% polyacrylamide gels (Laemmli, 1970) in the presence of $50 \mu \mathrm{M}$ phos-tag-acrylamide (Fujifilm Wako Chemicals)(Kinoshita et al., 2006). The in vitro 
phosphorylation of CDK1 by scCAK1 and hsCDK7 is described in detail in Figure 2 and Figure

3. The in vitro phosphorylation of CENP-T ${ }^{2-373}{ }^{2}$ by by CD1:Cyclin-B and CDK1:Cyclin-B:CKS1 complexes is described in detail in Figure 3 and Figure 4.

\section{Mass spectrometry of CDK1}

To analyze the phosphorylation of CDK1 by mass spectrometry, samples were reduced, alkylated and digested with LysC and Trypsin and prepared as previously described (Rappsilber et al., 2007). Obtained peptides were separated on an U3000 nanoHPLC system (Thermo Fisher Scientific). Samples were injected onto a desalting cartridge, desalted for 5 min using water in $0.1 \%$ formic acid, backflushed onto a Pepmap C18 nanoHPLC column (Thermo Fisher Scientific) and separated using a gradient from 5-30\% acetonitrile with $0.1 \%$ formic acid and a flow rate of 300 $\mathrm{nl} / \mathrm{min}$. Samples were directly sprayed via a nano-electrospray source in an Orbitrap type mass spectrometer (Thermo Fisher Scientific). The mass spectrometer was operated in a data-dependent mode acquiring one survey scan and subsequently up to fifteen MS/MS scans. To identify phospho-sites, the resulting raw files were processed with MaxQuant (version 1.6.17.0) searching for CDK1, Cyclin-B, and CKS1 sequences with acetylation (N-term), oxidation (M) and phosphorylation (STY) as variable modifications and carbamidomethylation (C) as fixed modification. A false discovery rate cut off of $1 \%$ was applied at the peptide and protein levels and as well on the site decoy fraction (Cox and Mann, 2008).

\section{Structures}

Surface views of CDK1:Cyclin-B:CKS1 (PDB 4YC3) (Brown et al., 2015)and of CDK2:Cyclin-A with bound ATP (PDB 1JST) (Russo et al., 1996) were prepared using Chimera X (version 0.9) (Goddard et al., 2018). 


\section{Reagent Availability}

The following plasmids are available through Addgene (addgene.org/Andrea_Musacchio/): pLIBGST-3C-CDK1 (177011), pLIB-HIS-TEV-Cyclin-B (177012), pLIB- HIS-TEV-CKS1(177013), and pLIB- HIS-TEV-scCAK1(177014). All other reagents are available upon request.

\section{Author Contributions}

CRediT contributorship: Conceptualization PH, AM; Formal Analysis PH; Funding acquisition AM; Investigation PH, FM, PJ; Methodology PH, SW, CK; Project Administration PH; Resources SW, CK; Supervision PH, AM; Visualization PH; Writing original draft PH; Writing - review \& editing PH, AM.

\section{Acknowledgements}

We thank David Barford for providing an scCAK1 expression plasmid, Priyanka Singh for early experiments with recombinant CDK1:Cyclin-B, and Kai Walstein for the preparation of TMR labeled CENP-T. AM gratefully acknowledges funding through the European Research Council Synergy Grant SyG 951430 BIOMECANET. 


\section{References}

Abdella R, Talyzina A, Chen S, Inouye CJ, Tjian R, He Y. 2021. Structure of the human Mediator-bound transcription preinitiation complex. Science. doi:10.1126/science.abg3074

Brown NR, Korolchuk S, Martin MP, Stanley WA, Moukhametzianov R, Noble MEM, Endicott JA. 2015. CDK1 structures reveal conserved and unique features of the essential cell cycle CDK. Nat Commun 6. doi:10.1038/ncomms7769

Chen X, Yin X, Li J, Wu Z, Qi Y, Wang X, Liu W, Xu Y. 2021. Structures of the human Mediator and Mediator-bound preinitiation complex. Science. doi:10.1126/science.abg0635

Cox J, Mann M. 2008. MaxQuant enables high peptide identification rates, individualized p.p.b.range mass accuracies and proteome-wide protein quantification. Nat Biotechnol 26:13671372. doi:10.1038/nbt.1511

Daub H, Olsen JV, Bairlein M, Gnad F, Oppermann FS, Körner R, Greff Z, Kéri G, Stemmann O, Mann M. 2008. Kinase-Selective Enrichment Enables Quantitative Phosphoproteomics of the Kinome across the Cell Cycle. Mol Cell 31:438-448. doi:10.1016/j.molcel.2008.07.007

Espinoza FH, Farrell A, Erdjument-Bromage H, Tempst P, Morgan DO. 1996. A cyclindependent kinase-activating kinase $(\mathrm{CAK})$ in budding yeast unrelated to vertebrate CAK. Science 273:1714-1717. doi:10.1126/science.273.5282.1714

Fisher RP, Morgan DO. 1994. A novel cyclin associates with M015/CDK7 to form the CDKactivating kinase. Cell 78:713-724. doi:10.1016/0092-8674(94)90535-5

Gelens L, Qian J, Bollen M, Saurin AT. 2018. The Importance of Kinase-Phosphatase Integration: Lessons from Mitosis. Trends Cell Biol 28:6-21. doi:10.1016/j.tcb.2017.09.005

Goddard TD, Huang CC, Meng EC, Pettersen EF, Couch GS, Morris JH, Ferrin TE. 2018. UCSF ChimeraX: Meeting modern challenges in visualization and analysis. Protein Sci 27:14-25. doi:10.1002/pro.3235

Greber BJ, Perez-Bertoldi JM, Lim K, Iavarone AT, Toso DB, Nogales E. 2020. The cryoelectron microscopy structure of the human CDK-activating kinase. Proc Natl Acad Sci U S A 117:22849-22857. doi:10.1073/pnas.2009627117

Hagopian JC, Kirtley MP, Stevenson LM, Gergis RM, Russo AA, Pavletich NP, Parsons SM, Lew J. 2001. Kinetic Basis for Activation of CDK2/Cyclin A by Phosphorylation*. J Biol Chem 276:275-280. doi:10.1074/jbc.M007337200

Hashimoto Y, Zhang Sheng, Zhang Shiying, Chen Y-R, Blissard GW. 2012. Erratum to: BTITnao38, a new cell line derived from Trichoplusia ni, is permissive for AcMNPV infection and produces high levels of recombinant proteins. BMC Biotechnol 12:12. doi:10.1186/1472-6750-12-12

Hegemann B, Hutchins JR, Hudecz O, Novatchkova M, Rameseder J, Sykora MM, Liu S, Mazanek M, Lenart P, Heriche JK, Poser I, Kraut N, Hyman AA, Yaffe MB, Mechtler K, Peters JM. 2011. Systematic phosphorylation analysis of human mitotic protein complexes. Sci Signal 4:rs12. doi:4/198/rs12 [pii] 10.1126/scisignal.2001993

Hermand D, Pihlak A, Westerling T, Damagnez V, Vandenhaute J, Cottarel G, Mäkelä TP. 1998. Fission yeast Csk1 is a CAK-activating kinase (CAKAK). EMBO J 17:7230-7238. doi:10.1093/emboj/17.24.7230

Hirakawa H, Ishikawa S, Nagamune T. 2015. Ca2+-independent sortase-A exhibits high selective protein ligation activity in the cytoplasm of Escherichia coli. Biotechnol J 10:1487-1492. doi:10.1002/biot.201500012

Hochegger H, Takeda S, Hunt T. 2008. Cyclin-dependent kinases and cell-cycle transitions: does one fit all? Nat Rev Mol Cell Biol 9:910-916. doi:10.1038/nrm2510 
Huis in 't Veld PJ, Jeganathan S, Petrovic A, Singh P, John J, Krenn V, Weissmann F, Bange T, Musacchio A. 2016. Molecular basis of outer kinetochore assembly on CENP-T. elife $\mathbf{5}$. doi:10.7554/eLife.21007

Huis in 't Veld PJ, Volkov VA, Stender ID, Musacchio A, Dogterom M. 2019. Molecular determinants of the Ska-Ndc80 interaction and their influence on microtubule tracking and force-coupling. eLife 8:e49539. doi:10.7554/eLife.49539

Jeffrey PD, Russo AA, Polyak K, Gibbs E, Hurwitz J, Massagué J, Pavletich NP. 1995. Mechanism of CDK activation revealed by the structure of a cyclinA-CDK2 complex. Nature 376:313-320. doi:10.1038/376313a0

Kaldis P, Sutton A, Solomon MJ. 1996. The Cdk-activating kinase (CAK) from budding yeast. Cell 86:553-564. doi:10.1016/s0092-8674(00)80129-4

Kim S, Yu H. 2015. Multiple assembly mechanisms anchor the KMN spindle checkpoint platform at human mitotic kinetochores. J Cell Biol 208:181-96. doi:10.1083/jcb.201407074

Kinoshita E, Kinoshita-Kikuta E, Takiyama K, Koike T. 2006. Phosphate-binding tag, a new tool to visualize phosphorylated proteins. Mol Cell Proteomics MCP 5:749-757. doi:10.1074/mcp.T500024-MCP200

Kõivomägi M, Örd M, Iofik A, Valk E, Venta R, Faustova I, Kivi R, Balog ERM, Rubin SM, Loog M. 2013. Multisite phosphorylation networks as signal processors for Cdk1. Nat Struct Mol Biol 20:1415-1424. doi:10.1038/nsmb.2706

Kõivomägi M, Valk E, Venta R, Iofik A, Lepiku M, Balog ER, Rubin SM, Morgan DO, Loog M. 2011. Cascades of multisite phosphorylation control Sic1 destruction at the onset of S phase. Nature 480:128-31. doi:10.1038/nature10560

Laemmli UK. 1970. Cleavage of Structural Proteins during the Assembly of the Head of Bacteriophage T4. Nature 227:680. doi:10.1038/227680a0

Larochelle S, Pandur J, Fisher RP, Salz HK, Suter B. 1998. Cdk7 is essential for mitosis and for in vivo Cdk-activating kinase activity. Genes Dev 12:370-381. doi:10.1101/gad.12.3.370

Morgan DO. 2007. The Cell Cycle: Principles of Control. New Science Press.

Nishino T, Rago F, Hori T, Tomii K, Cheeseman IM, Fukagawa T. 2013. CENP-T provides a structural platform for outer kinetochore assembly. EMBO J 32:424-36. doi:10.1038/emboj.2012.348

Olsen JV, Vermeulen M, Santamaria A, Kumar C, Miller ML, Jensen LJ, Gnad F, Cox J, Jensen TS, Nigg EA, Brunak S, Mann M. 2010. Quantitative phosphoproteomics reveals widespread full phosphorylation site occupancy during mitosis. Sci Signal 3:ra3. doi:10.1126/scisignal.2000475

Piano V, Alex A, Stege P, Maffini S, Stoppiello GA, Huis in 't Veld PJ, Vetter IR, Musacchio A. 2021. CDC20 assists its catalytic incorporation in the mitotic checkpoint complex. Science 371:67-71. doi:10.1126/science.abc1152

Rago F, Gascoigne KE, Cheeseman IM. 2015. Distinct organization and regulation of the outer kinetochore KMN network downstream of CENP-C and CENP-T. Curr Biol CB 25:671677. doi:10.1016/j.cub.2015.01.059

Rappsilber J, Mann M, Ishihama Y. 2007. Protocol for micro-purification, enrichment, prefractionation and storage of peptides for proteomics using StageTips. Nat Protoc 2:1896906. doi:10.1038/nprot.2007.261

Russo AA, Jeffrey PD, Pavletich NP. 1996. Structural basis of cyclin-dependent kinase activation by phosphorylation. Nat Struct Biol 3:696-700. doi:10.1038/nsb0896-696

Saiz JE, Fisher RP. 2002. A CDK-activating kinase network is required in cell cycle control and transcription in fission yeast. Curr Biol CB 12:1100-1105. doi:10.1016/s0960-

9822(02)00903-x 
bioRxiv preprint doi: https://doi.org/10.1101/2021.09.24.461690; this version posted September 24, 2021 . The copyright holder for this

preprint (which was not certified by peer review) is the author/funder, who has granted bioRxiv a license to display the preprint in perpetuity. It is made available under aCC-BY-NC 4.0 International license.

Singh P, Pesenti ME, Maffini S, Carmignani S, Hedtfeld M, Petrovic A, Srinivasamani A, Bange T, Musacchio A. 2021. BUB1 and CENP-U, Primed by CDK1, Are the Main PLK1 Kinetochore Receptors in Mitosis. Mol Cell. doi:10.1016/j.molcel.2020.10.040

Thuret J-Y, Valay J-G, Faye G, Mann C. 1996. Civ1 (CAK In Vivo), a Novel Cdk-Activating Kinase. Cell 86:565-576. doi:10.1016/S0092-8674(00)80130-0

Trowitzsch S, Bieniossek C, Nie Y, Garzoni F, Berger I. 2010. New baculovirus expression tools for recombinant protein complex production. J Struct Biol. doi:S1047-8477(10)00057-2 [pii] 10.1016/j.jsb.2010.02.010

Weissmann F, Petzold G, VanderLinden R, Huis in 't Veld PJ, Brown NG, Lampert F, Westermann S, Stark H, Schulman BA, Peters JM. 2016. biGBac enables rapid gene assembly for the expression of large multisubunit protein complexes. Proc Natl Acad Sci U A 113:E2564-9. doi:10.1073/pnas.1604935113

Wood DJ, Endicott JA. 2018. Structural insights into the functional diversity of the CDK-cyclin family. Open Biol 8. doi:10.1098/rsob.180112

Yu J, Raia P, Ghent CM, Raisch T, Sadian Y, Cavadini S, Sabale PM, Barford D, Raunser S, Morgan DO, Boland A. 2021. Structural basis of human separase regulation by securin and CDK1-cyclin B1. Nature 596:138-142. doi:10.1038/s41586-021-03764-0

Zhang S, Chang L, Alfieri C, Zhang Z, Yang J, Maslen S, Skehel M, Barford D. 2016. Molecular mechanism of APC/C activation by mitotic phosphorylation. Nature 533:260-264. doi:10.1038/nature17973 
bioRxiv preprint doi: https://doi.org/10.1101/2021.09.24.461690; this version posted September 24, 2021. The copyright holder for this

preprint (which was not certified by peer review) is the author/funder, who has granted bioRxiv a license to display the preprint in perpetuity. It is made available under aCC-BY-NC 4.0 International license.

\section{Supplementary Table 1: Phosphorylation in CDK1:Cyclin-B:CKS1}

See Figure 3 and Supplementary Figure 1 for an in-depth analysis of the kinase activity of this sample (CCC) and the phosphorylation of Thr161 in CDK1.
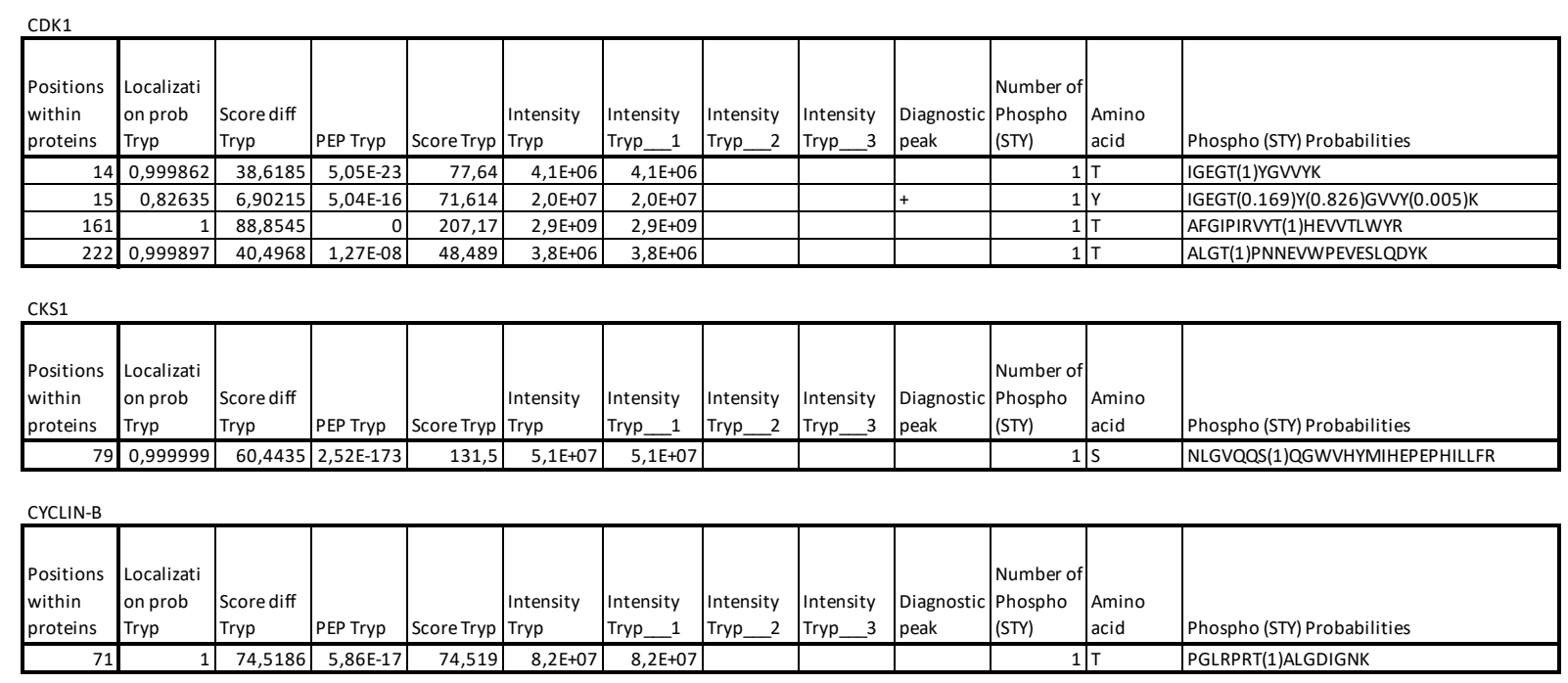